This item was submitted to Loughborough's Institutional Repository (https://dspace.lboro.ac.uk/) by the author and is made available under the following Creative Commons Licence conditions.

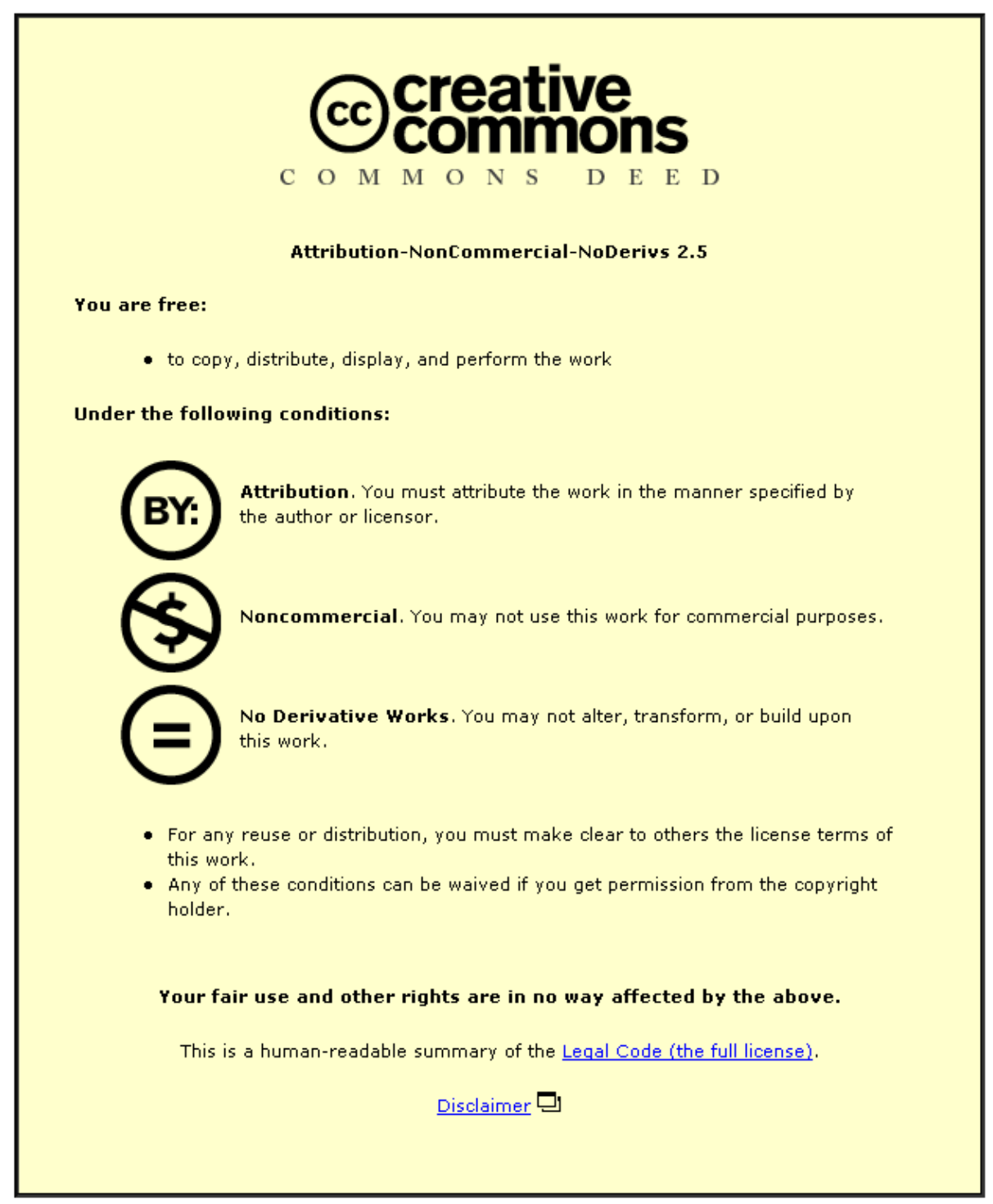

For the full text of this licence, please go to: http://creativecommons.org/licenses/by-nc-nd/2.5/ 


\title{
Variable Tap-Length Adaptive Algorithm Which Exploits both Second and Fourth Order Statistics
}

\author{
Yonggang Zhang, Leilei Li and Jonathon A. Chambers \\ Advanced Signal Processing Group, Department of Electronic \& Electrical Engineering \\ Loughborough University, Loughborough LE11 3TU, UK. Email: y.zhang5@lboro.ac.uk
}

\begin{abstract}
A new variable tap-length adaptive algorithm which exploits both second and fourth order statistics is proposed in this paper. In this algorithm, the tap-length of the adaptive filter is varying rather than fixed, and controlled by fourth order statistics, whereas the coefficient update retains a conventional least mean square (LMS) form. As will be seen in the simulation results, the proposed algorithm has a faster convergence rate as compared with an existing variable tap-length LMS algorithm which is based only on second order statistics in sub-Gaussian noise environments.
\end{abstract}

Keywords: Adaptive filters, LMS algorithm, variable tap-length, fourth order statistics.

\section{INTRODUCTION}

The LMS adaptive algorithm has been extensively used as a consequence of its simplicity and robustness [1][2]. In many applications of the LMS algorithm, the tap-length of the adaptive filter is kept fixed. However, in certain situations the tap-length of the optimal filter is unknown or variable. According to the analysis in [3][4], the mean square error (MSE) of the adaptive filter is likely to increase if the tap-length is under-modelled. To avoid such a situation, a sufficiently large filter taplength is needed. However, the computational cost and the excess mean square error (EMSE) of the LMS algorithm will increase if the tap-length is too large, thus a variable tap-length LMS algorithm is needed to find a proper choice of the tap-length.

Several variable tap-length LMS algorithms have been proposed in recent years [3][5][6][7][8][9]; a summary of these works is given in [10]. As analyzed in [10], the fractional tap-length (FT) algorithm is more robust and has lower computational complexity when compared with other methods. A convex combination structure of the FT algorithm has been proposed in [11] to establish the optimal tap-length in high noise conditions, in which two filters are updated simultaneously with different parameters, so that the overall filter can obtain both a rapid convergence rate from the fast filter and a smooth curve for the steady-state tap-length from the slow filter. A steady state performance analysis of the FT algorithm is provided in [12] and it also gives a guideline for the parameter choice of the FT algorithm.

All the above work on the update of both the adaptive filter coefficients and the tap-length of the LMS al- gorithm are based on second order statistics (SOS). It is well known that algorithms based on higher order statistics (HOS) potentially work more efficiently for sub-Gaussian noise environments since they utilize the information contained in higher order moments, which yields a better approximation of the actual distribution of the signal. A typical algorithm is the least mean fourth (LMF) algorithm, which has been proved to have a faster convergence rate as compared with the LMS algorithm [13] in sub-Gaussian noise environments. However, it suffers from a stability condition. If the LMF algorithm is initialized far from the optimal filter coefficients, it may be unstable [14].

In this paper, we propose a new variable tap-length adaptive algorithm, in which the update of the tap-length is controlled by fourth order statistics whilst the coefficient update retains as conventional LMS form. By utilizing such an approach, we utilize the good properties of both SOS and HOS, i.e., good stability and quick convergence. As will be shown by simulations, the proposed approach has a faster convergence rate as compared with the FT variable tap-length LMS algorithm in sub-Gaussian noise environments.

The remainder of the paper is organized as follows: In Section 2 we formulate the FT variable tap-length LMS algorithm [10]. The proposed algorithm is given in Section 3. Simulations are performed in Section 4 to show the good properties of the proposed algorithm. Section 5 concludes the paper.

\section{FT VARIABLE TAP-LENGTH LMS ALGORITHM}

The FT variable tap-length LMS algorithm is designed to find the optimal filter tap-length. In agreement with most approaches used to derive algorithms for adaptive filtering the design problem is related to the optimization of a certain criterion that is dependent on the tap-length. For convenience, we shall formulate the LMS algorithm within a system identification framework, in which the unknown filter $\mathbf{c}_{L_{o p t}}$ has an unknown tap-length $L_{\text {opt }}$ which is to be identified. In this model, the desired signal $d(n)$ is represented as

$$
d(n)=\mathbf{x}_{L_{o p t}}^{T}(n) \mathbf{c}_{L_{o p t}}+v(n)
$$

where $\mathbf{x}_{L_{o p t}}(n)$ is the input vector with a tap length of $L_{o p t}, v(n)$ is a zero mean additive noise term uncorrelated with the input, $n$ denotes the discrete time index, 
and $(\cdot)^{T}$ denotes the transpose operation. In this work all quantities are assumed to be real valued.

For convenience of description we assume that at steady state the tap-length of the adaptive filter is a fixed value and denoted by $L ; \mathbf{w}_{L}$ and $\mathbf{x}_{L}(n)$ are respectively the corresponding steady-state adaptive filter vector and input vector. Also, we define the segmented steady-state error as [10]

$$
e_{M}^{(L)}(n)=d(n)-\mathbf{w}_{L, 1: M}^{T} \mathbf{x}_{L, 1: M}(n), \quad \text { as } \mathrm{n} \rightarrow \infty
$$

where $1 \leq M \leq L, \mathbf{w}_{L, 1: M}$ and $\mathbf{x}_{L, 1: M}(n)$ are respectively vectors consisting of the first $M$ coefficients of the steady-state filter vector $\mathbf{w}_{L}$ and the input vector $\mathbf{x}_{L}(n)$. The mean square of this segmented steady-state error is defined as $\xi_{M}^{(L)}=E\left\{\left(e_{M}^{(L)}(n)\right)^{2}\right\}$. The underlying basis of the FT method is to find the minimum value of $L$ satisfying [10]:

$$
\xi_{L-\Delta}^{(L)}-\xi_{L}^{(L)} \leq \varepsilon
$$

where $\Delta$ is a positive integer less than $L$ and $\varepsilon$ is a small positive value determined by the system requirements. The minimum $L$ that satisfies (3) is then chosen as the optimal tap-length. A detailed description of this criterion and another similar criterion can be found in [10].

Gradient-based methods can be used to estimate $L$ on the basis of (3). However, the tap length that should be used in the adaptive filter structure must be an integer, and this constrains the adaptation of the tap length. Different approaches have been applied to solve this problem [6][7][8][9][10]. In [10], the concept of "pseudo fractional tap-length", denoted by $l_{f}(n)$, is utilized to make instantaneous tap-length adaptation possible. The update of the fractional tap-length is as follows:

$l_{f}(n+1)=\left(l_{f}(n)-\alpha\right)-\gamma\left[\left(e_{L(n)}^{(L(n))}\right)^{2}-\left(e_{L(n)-\Delta}^{(L(n))}\right)^{2}\right]$

where $\gamma$ is the step size for the tap-length adaptation, and $\alpha$ is a positive leakage parameter [10]. As explained in [10], $l_{f}(n)$ is no longer constrained to be an integer, and the tap-length $L(n+1)$, which will be used in the adaptation of the filter weights in the next iteration, is obtained from the fractional tap-length $l_{f}(n)$ as follows:

$$
L(n+1)= \begin{cases}\left\lfloor l_{f}(n)\right\rfloor & \text { if }\left|L(n)-l_{f}(n)\right|>\delta \\ L(n) & \text { otherwise }\end{cases}
$$

where $\lfloor$.$\rfloor is the floor operator, which rounds down the$ embraced value to the nearest integer and $\delta$ is a small integer.

In order to speed up the convergence rate of the FT variable tap-length LMS algorithm, the step size is made variable rather than fixed, according to the range of $\mu$ described in [3]:

$$
\mu(n)=\mu^{\prime} /\left((L(n)+2) \sigma_{x}^{2}\right)
$$

where $\mu^{\prime}$ is a constant and $\sigma_{x}^{2}$ is the variance of the input. The coefficients of the adaptive filter are then updated according to the LMS algorithm by using $L(n)$ and $\mu(n)$.
As analyzed in [12] the parameter $\gamma$ controls the adaptation process of the variable tap-length. Similar to the step size in the LMS algorithm, a large parameter $\gamma$ will speed up the convergence rate of the tap-length, but will result in a large fluctuation of the steady-state tap-length. Once the tap length fluctuates under the optimal tap-length, extra error will be introduced. This is named as the undermodelling phenomenon for variable tap-length algorithms. On the other hand, a small parameter $\gamma$ can obtain a small fluctuation of the steady-state tap-length, but lead to a slow convergence rate of both the tap-length and excess mean square error (EMSE).

Motivated by the FT algorithm, next we will describe a new variable tap-length LMS algorithm.

\section{PROPOSED ALGORITHM}

It is clear to see from (4) that in the FT algorithm, the tap-length of the adaptive filter is updated based on the information provided by SOS, i.e., squared value of instantaneous errors, and no HOS information is utilized. In the proposed algorithm, the update of the coefficients of the adaptive filters still utilizes the SOS, i.e., the LMS algorithm, to have a good stability property, but the update of the tap-length is driven by HOS to obtain a faster convergence rate. The update of the tap-length of the proposed algorithm is as follows

$l_{f}(n+1)=\left(l_{f}(n)-\alpha\right)-\gamma\left[\left(e_{L(n)}^{(L(n))}\right)^{4}-\left(e_{L(n)-\Delta}^{(L(n))}\right)^{4}\right]$

It is clear to see that the update equation (7) is very similar to (4), and the fourth moments of the instantaneous errors are utilized. Similar to that in [10] the tap-length $L(n+1)$ which will be used in the adaptation of the filter weights in the LMS algorithm is obtained from the fractional tap-length $l_{f}(n)$ according to (5), and the step size is chosen according to (6), followed by the update of the adaptive filter coefficients according to the LMS algorithm.

By rewriting $\gamma\left[\left(e_{L(n)}^{(L(n))}\right)^{4}-\left(e_{L(n)-\Delta}^{(L(n))}\right)^{4}\right]$ in (7) as
$\left[\left(e_{L(n)}^{(L(n))}\right)^{2}+\left(e_{L(n)-\Delta}^{(L(n))}\right)^{2}\right]\left[\left(e_{L(n)}^{(L(n))}\right)^{2}-\left(e_{L(n)-\Delta}^{(L(n))}\right)^{2}\right]$ we can find that the proposed algorithm can be deemed as the FT algorithm with a variable parameter $\gamma\left[\left(e_{L(n)}^{(L(n))}\right)^{2}+\left(e_{L(n)-\Delta}^{(L(n))}\right)^{2}\right]$. As compared with a fixed parameter $\gamma$, it is large initially and small at steady state. Thus as compared with the FT algorithm, the proposed algorithm will have a smaller variance of the tap-length but a similar convergence rate of the tap-length, which results in a quick convergence rate of the EMSE, but also reduces or avoids the under-modelling phenomenon. As will also be shown in the simulations in the next section, the proposed algorithm has a better performance as compared with the FT algorithm in sub-Gaussian noise environments. 

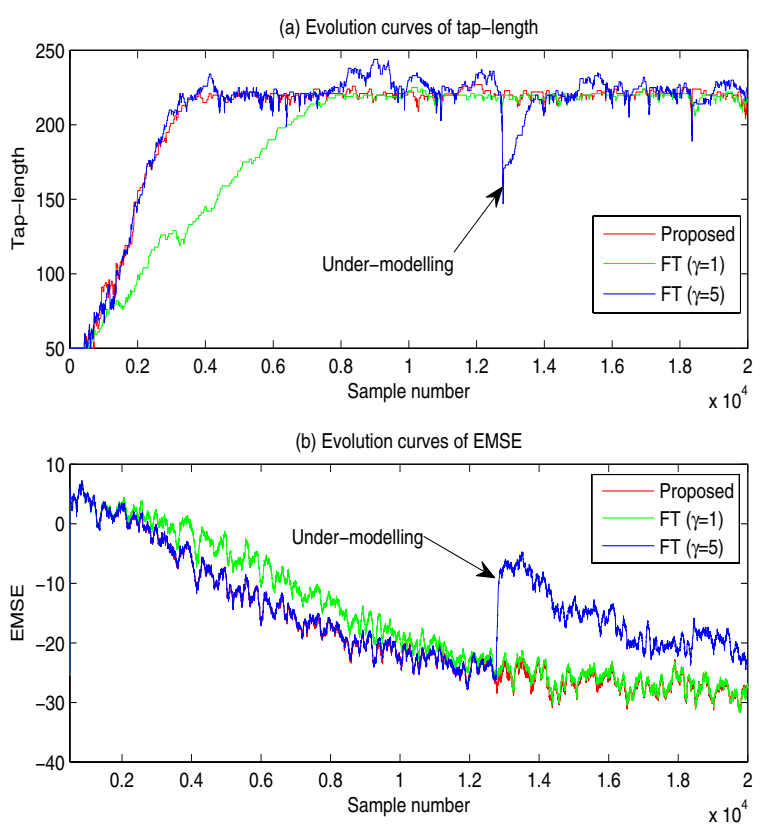

Fig. 1. The evolution curves of the tap-length and EMSE for both the proposed algorithm and the FT algorithm with a uniformly distributed noise and $\mathrm{SNR}=0 \mathrm{~dB}$.

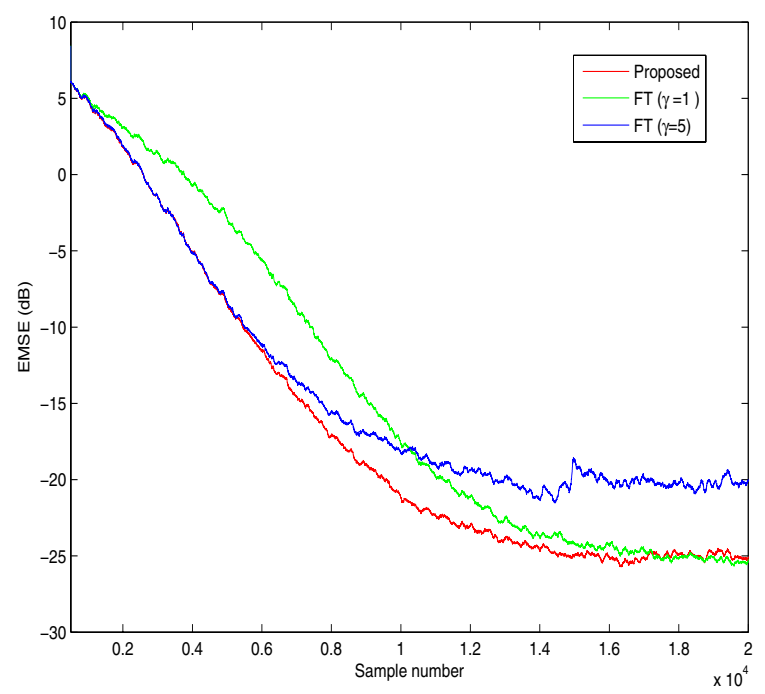

Fig. 2. The evolution curves of the EMSE for both the proposed algorithm and the FT algorithm with a uniformly distributed noise and $\mathrm{SNR}=0 \mathrm{~dB}$, obtained by averaging 100 independent runs.

\section{SIMULATIONS}

Two simulations are performed in this section to show the advantages of the proposed algorithm as compared with the FT algorithm in sub-Gaussian noise environments. The setup of the first simulation is as follows. The impulse response sequence of the unknown filter is
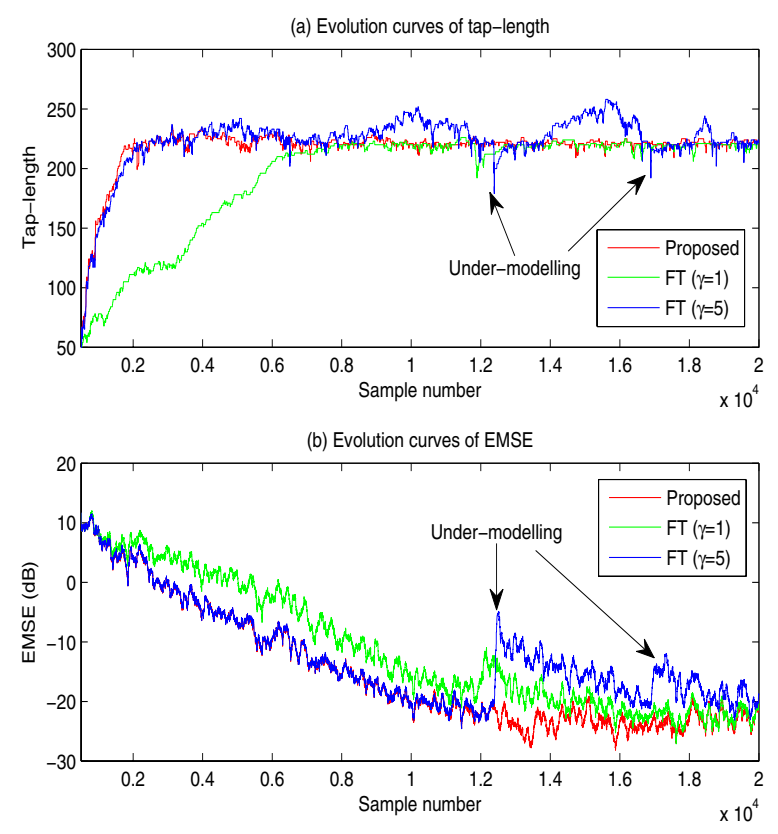

Fig. 3. The evolution curves of the tap-length and EMSE for both the proposed algorithm and the FT algorithm with a binary noise sequence and $\mathrm{SNR}=0 \mathrm{~dB}$.

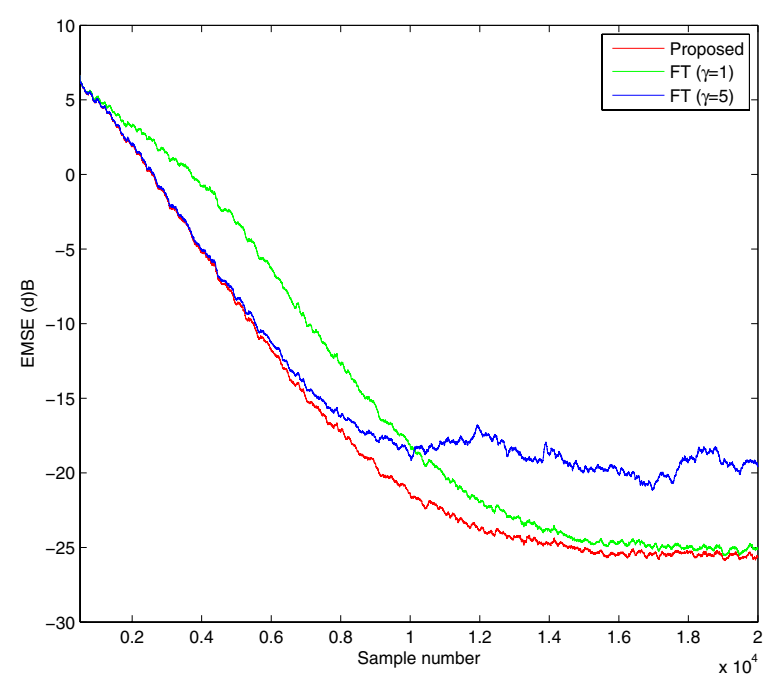

Fig. 4. The evolution curves of the EMSE for both the proposed algorithm and the FT algorithm with a binary noise sequence and $\mathrm{SNR}=0 \mathrm{~dB}$, obtained by averaging 100 independent runs.

a random sequence with zero mean and variance 0.01 . The tap-length $L_{o p t}$ is set to 200 . The input signal is a white Gaussian sequence with zero mean and unit variance. The noise signal is a white uniformly distributed sequence with zero mean and scaled to make the SNR OdB. The parameter $\delta$ in (5) is set to 2 . The step size $\mu^{\prime}$ in (6) is set to 0.5 . The leakage parameter $\alpha$ is set to 0.01 , 
and $\Delta$ is set to 20 .

To show the advantages of the proposed algorithm, we perform the FT algorithm with different values $\gamma=1$ and $\gamma=5$ respectively. The parameter $\gamma$ for the proposed algorithm is set to 0.2 .

In Fig. 1, which is best viewed in colour, we plot one run of the simulation according to the above set up. It is clear to see in Fig. 1(a) that the tap-length of the proposed algorithm has a similar convergence rate as that of the FT algorithm with a parameter $\gamma=5$, but has a much smaller steady state variance. For the FT algorithm with a large parameter $\gamma=5$, the under-modelling phenomenon appears, which results in an increase of the EMSE, as can be seen in Fig. 1(b). The proposed algorithm has both a quicker convergence of the tap-length and the EMSE as compared with that of the FT algorithm with $\gamma=1$.

In Fig. 2 we plot the evolution curves of the EMSE of both algorithms by averaging the results of 100 independent runs. It is clear to see from this figure that the proposed algorithm has a similar convergence rate of the EMSE with that of the FT algorithm with $\gamma=5$, but approximately 8dB EMSE improvement. It has a similar steady state EMSE with that of the FT algorithm with $\gamma=1$ but a quicker convergence. Based on the above simulation results we conclude that the proposed algorithm outperforms the FT algorithm in this uniform distributed noise environment.

In the second simulation, the set up is the same as that of the first simulation, but the noise is a binary sequence and scaled to make the SNR OdB. Similar to that of the first simulation, we plot the evolution curves of the tap-length and EMSE obtained from a single run of both the proposed algorithm and the FT algorithm in Fig. 3. In Fig. 4 the evolution curves of both algorithms are obtained by averaging the results of 100 independent runs. Again, it is clear to see from both Fig. 3 and Fig. 4 that the proposed algorithm outperforms the FT algorithm for the binary noise environment.

\section{CONCLUSION}

A new variable tap-length adaptive algorithm is proposed in this paper. In the algorithm, the update of the taplength is controlled by fourth order statistics. As have been shown by simulation results the proposed algorithm has a better performance as compared with the FT variable tap-length LMS algorithm in sub-Gaussian noise environments, and can be potentially utilized in many applications.

\section{REFERENCES}

[1] B. Farhang-Boroujeny, Adaptive Filters: Theory and Applications, New York, Wiley, 1998.

[2] A. H. Sayed, Fundamentals of Adaptive Filtering, New York, Wiley, NJ, 2003.
[3] Y. Gu, K. Tang, H. Cui, and W. Du, "Convergence analysis of a deficient-length LMS filter and optimal-length sequence to model exponential decay impulse response," IEEE Signal Processing Lett., vol. 10, no. 1, pp. 4-7, Jan. 2003.

[4] K. Mayyas, "Performance analysis of the deficient length LMS adaptive algorithm," IEEE Trans. Signal Processing, vol. 53, no. 8, pp. 2727-2734, Aug. 2005.

[5] Y. Zhang, J. A. Chambers, S. Sanei, P. Kendrick and T. J. Cox, "A new variable tap-length LMS algorithm to model an exponential decay impulse response," IEEE Signal Processing Lett., vol. 14, no. 4, pp. 263-266, Apr. 2007.

[6] F. Riero-Palou, J. M. Noras, and D. G. M. Cruickshank, "Linear equalisers with dynamic and automatic length selection," Electronics Lett., vol. 37, no. 25, pp. 1553-1554, Dec. 2001.

[7] Y. Gu, K. Tang, H. Cui, and W. Du, "LMS algorithm with gradient descent filter length," IEEE Signal Processing Lett., vol. 11, no. 3, pp. 305-307, Mar. 2004.

[8] Y. Gong and C. F. N. Cowan, "A novel variable tap-length algorithm for linear adaptive filters," in Proc. ICASSP2004, Jan. 2004.

[9] Y. Gong and C. F. N. Cowan, "Structure adaptation of linear MMSE adaptive filters," Proc. Inst. Elect. Eng.-Vision, Image, Signal Processing, vol. 151, no. 4, pp. 271-277, Aug. 2004.

[10] Y. Gong and C. F. N. Cowan, "An LMS style variable tap-length algorithm for structure adaptation," IEEE Trans. Signal Processing, vol. 53, no. 7, pp. 2400-2407, July 2005.

[11] Y. Zhang and J. A. Chambers, "Convex combination of adaptive filters for variable tap-length LMS algorithm," IEEE Signal Processing Lett., vol. 13, no. 10 , pp. 628-631, Oct. 2006.

[12] Y. Zhang, J. A. Chambers, S. Sanei, P. Kendrick and T. J. Cox, "Steady-state performance analysis of a variable tap-length LMS algorithm," IEEE Tran. Signal Processing, vol. 56, no. 2, pp. 839-845, Feb. 2008.

[13] E. Walach and B. Widrow, "The least mean fourth (LMF) adaptive algorithm and its famiiy," IEEE Trans. Information Theory, vol. 30, pp. 275-283, July 1984.

[14] P. I. Hbscher, J. C. M. Bermudez and V. H. Nascimento, "A mean-square stability analysis of the least mean forth adaptive algorithm," IEEE Trans. Signal Processing, vol. 55, no. 8, pp. 4018-4028, Aug. 2007. 